\title{
Adult onset scapuloperoneal myopathy: diagnostic value of nerve morphometry and multiple muscle biopsies
}

\author{
W C YEE, A F HAHN, J J GILBERT \\ From the Departments of Clinical Neurological Sciences and of Pathology, Victoria Hospital, The University of \\ Western Ontario, London, Ontario, Canada
}

SUMMARY In the scapuloperoneal syndrome, differentiation between neurogenic and myopathic processes may be difficult despite electromyography and muscle biopsy. Extensive analysis, including morphometry, was conducted on multiple nerve and muscle biopsies from two adult onset, sporadic cases with the syndrome. These studies confirm a myopathic process and further define the entity of adult onset scapuloperoneal myopathy.

The scapuloperoneal syndrome is a rare entity characterised clinically by the combination of proximal shoulder girdle and distal lower limb muscle wasting and weakness. The entity is probably heterogenous and has been related to primary myopathic and to neurogenic disease processes. ${ }^{1-3}$ However, in spite of EMG and nerve conduction studies and muscle biopsy, elucidation of the underlying disease process is often difficult because of non-specific or contradictory results. ${ }^{245}$

We investigated two patients with the syndrome with adult onset and negative family histories. Extensive studies of multiple muscle and nerve biopsies, which included light and electron microscopy, muscle histochemistry, teased nerve fibre analysis and nerve fibre morphometry, were performed. These studies helped to confirm a primary myopathic disease process. The findings in these patients further define the entity of adult onset scapuloperoneal myopathy. ${ }^{167}$

\section{Methods}

Muscle biopsies were taken from a distal lower limb muscle, the tibialis anterior or the extensor hallucis longus, and from a proximal upper limb muscle, the deltoid. Cryostat sections were processed for enzyme histochemistry, haematoxylineosin and Gomori's trichrome stains. Fixed tissues were embedded in resin and semithin sections stained with

Address for reprint requests: Dr W C Yee, Rm 220, 770 Bannatyne Avenue, University of Manitoba, Winnipeg, Manitoba, Canada R3E OW3.

Received 14 August 1987 and in revised form 2 February 1988. Accepted 10 February 1988 toluidine blue were viewed by light microscopy. Ultrathin sections were viewed using a Philips 401 electron microscope.

Fascicular nerve biopsies were obtained from both superficial and deep peroneal nerves and processed using the method of Dyck. ${ }^{8}$ Semithin sections stained with toluidine blue were analysed by light microscopy. Ultrathin sections were viewed using a Philips 401 electron microscope. The teased fibre preparation of the deep peroneal nerves contained a minimum of 100 myelinated fibres. The fibres were analysed and classified according to Dyck. ${ }^{8}$ A morphometric analysis of myelinated fibre density and size distribution in the deep and superficial peroneal nerves was performed using the method of Dyck. ${ }^{8}$

\section{Case reports}

Case 1

This 64 year old man of Maltese origin first noted difficulty with walking in the snow at the age of 55 years. A physician noted bilateral foot drop at the time and diagnosed "muscular dystrophy." Two years later, weakness in lifting the arms was noted. These symptoms progressed insidiously over the years. Currently, he requires the use of a right ankle brace for foot drop. There is no difficulty getting up from a chair and hand grip remains strong. There was no family history of related muscular disorders.

On examination, he walked with bilateral foot drop. Cranial nerve examination was normal with no facial or neck muscle weakness. Winging of both scapulae was observed in association with moderate weakness and wasting of all shoulder girdle muscles. The biceps, triceps and forearm extensor muscles were mildly weak but other distal muscles, including the small muscles of the hands, were spared. In the lower limbs, severe wasting of the anterior compartment of the legs was associated with total loss of power in dorsiflexor and evertor muscles. The right extensor digitorum brevis 


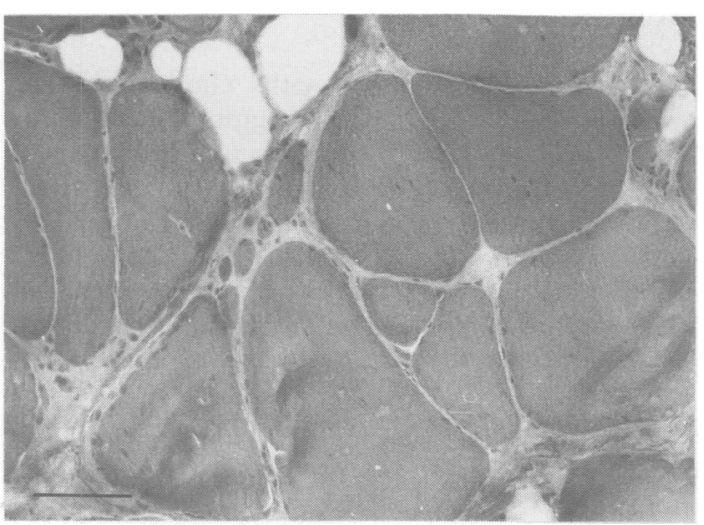

Fig 1 Deltoid, Case 1. Fibre size variation with hypertrophied and atrophied fibres, internal nuclei and fibre splitting. Gormori's Trichrome stain. (Bar $=70 \mu \mathrm{m})$.

muscle was wasted though the left retained more muscle bulk. Gastrocnemii muscles were mildly increased in bulk and had normal strength. Proximal muscles of the lower limbs were also of normal strength. Triceps, brachioradialis and ankle jerks were unobtainable. Knee jerks were diminished and biceps jerks preserved. Plantar responses were downgoing. The sensory examination was normal. There was no action or percussion myotonia. Serum creatine kinase was slightly elevated to $356 \mathrm{U} / 1$ (normal 36-188 U/1). Serum lactic dehydrogenase levels were normal. The EKG was normal.

\section{EMG and nerve conduction studies}

Motor conduction velocities and latencies were normal for the median, tibial and peroneal nerves. The right median and sensory conduction velocities and distal latencies were normal with normal sensory action potentials. A mild compressive palsy at the elbow was noted in nerve conduction studies of the right ulnar nerve. Needle EMG studies demonstrated numerous fibrillation potentials, positive sharp waves and complex high frequency discharges in the right tibialis anterior and extensor digitorum brevis muscles. There was an increase in polyphasic motor unit potentials of normal or short duration in both muscles. Needle EMGs of five upper limb muscles did not demonstrate any abnormality.

\section{Muscle biopsy}

Biopsy of the extensor hallucis longus muscle showed severe replacement by fat and connective tissue. A few hypertrophied muscle fibres remained. In the deltoid muscle biopsy, (fig 1), muscle fibre size varied with hypertrophied fibres and small fibres of both fibre types distributed

Table 1 Myelinated fibre densities of superficial and deep peroneal nerves (fibres $/ \mathrm{mm}^{2}$.)

\begin{tabular}{lll}
\hline & Deep peroneal nerve & Superficial peroneal nerve \\
\hline Case 1 & 7,613 & 11,669 \\
Case 2 & 8,891 & 10,176 \\
\hline
\end{tabular}

Table 2 Teased fibre analysis of deep peroneal nerve

\begin{tabular}{lllllllll}
\hline & \multirow{2}{*}{ No. } & & \multicolumn{6}{l}{ Classification (\%) } \\
\cline { 2 - 6 } \cline { 5 - 8 } & Fibres counted & & $A \& B$ & $C$ & $D$ & $E$ & $F$ \\
\hline Case 1 & 110 & 90 & 0 & 0 & 0.9 & 10 \\
Case 2 & 101 & 93 & 0 & 0 & 0 & 7 \\
\hline
\end{tabular}

(Classification according to Dyck ${ }^{8}$; A-normal appearance, Bmyelin irregularity and wrinkling, otherwise similar to $\mathrm{A}, \mathrm{C}-$ paranodal or internodal segmental demyelination, myelin thinning of $50 \%$ or more, $\mathrm{D}-$ same as $\mathrm{C}$, except myelin thinning $<50 \%$, $\mathrm{E}-$ rows of myelin ovoids, $\mathrm{F}$-excessive variation in myelin thickness between internodes without segmental demyelination)

randomly. Fibre splitting accounted for a few small groups of small fibres. Rare small angulated fibres were also evident. Internal nuclei were moderately prominent. Muscle fibre degeneration, necrosis and regeneration were not observed. Occasional fibres with ringbinden were noted on semithin and ultrathin microscopy. No inflammatory infiltrates were present.

\section{Nerve biopsy}

The light and electron microscopic appearances of both superficial and deep peroneal nerves were normal with no evidence of axonal loss, axonal degeneration, demyelination or remyelination. The myelinated fibre densities and fibre diameter distributions were normal (table 1, Fig 4a, b). Teased fibre analysis of the deep peroneal nerve was also normal for age (table 2).

\section{Case 2}

This 58 year old Caucasian woman (fig 2) was noted incidentally by her doctor to have weakness of the left leg 7 years earlier. She subsequently complained of progressive weakness in her legs and difficulty in lifting up her arms. There was a tendency to catch her toes on walking, and some difficulty rising from a chair. The patient was a known hypertensive on treatment with clonidine and aldactazide (spironolactone and hydrochlorothiazide). There was no known family history of muscle disease.

On examination, she was noted to have prominently sloping shoulders and bilateral foot drop. There was no facial muscle weakness. Mild neck flexor muscle weakness was noted. Winging and elevation of the scapulae were present together with moderate weakness and wasting in all shoulder girdle muscles, including the deltoid and biceps muscles. Triceps muscles and distal forearm and hand muscles were unaffected. In the lower limbs, there was mild weakness in proximal muscles but marked weakness and wasting were noted in the anterior compartment muscles of the legs with severe weakness in dorsiflexion and eversion. The gastrocnemii muscles appeared to be spared with normal plantar flexion. Deep tendon reflexes were normal with the exception of the ankle jerks, which were absent. No response was observed with the plantar reflex. The sensory examination was normal. There was no action or percussion myotonia.

Serum creatine kinase was minimally elevated to $207 \mathrm{U} / 1$ (normal 36-188 U/1). The lactic dehydrogenase level was similarly mildly elevated to $240 \mathrm{U} / 1$ (normal 100-225 U/1). The EKG showed sinus rhythm with minor non-specific repolarization changes. An echocardiogram showed no structural or functional abnormality. 


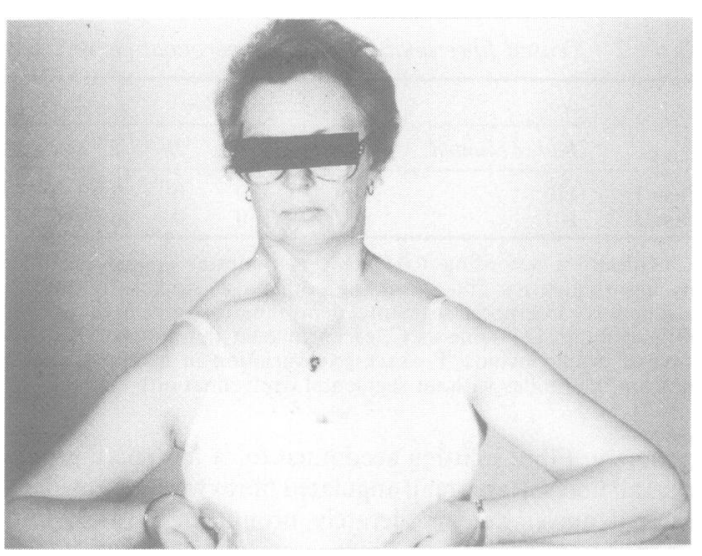

Fig 2 Case No. 2. Note elevation of scapula and wasting of deltoid muscle.

\section{EMG and nerve conduction studies}

Superficial peroneal and sural sensory conduction studies were normal with normal velocities, distal latencies and sensory action potentials. Peroneal motor conduction velocity was normal with a normal evoked amplitude recorded from the extensor digitorum brevis muscle. EMGs of the tibialis anterior, extensor digitorum brevis, quadriceps and deltoid muscles showed no abnormal spontaneous activity. In the tibialis anterior and extensor digitorum brevis muscles, the amplitude and duration of motor unit potentials were often increased with an increased firing rate. There was also an increased incidence of polyphasic units in these muscles.

\section{Muscle biopsy}

The tibialis anterior biopsy showed moderate infiltration by fat and connective tissue. Muscle fibre size variation was pronounced with atrophied and hypertrophied fibres in a random distribution. Small fibres were rounded. Internal nuclei were prominent. Necrotic, degenerating or regenerating fibres were not observed. Frequent ringbinden were seen and on electron microscopy, some areas of focal myofibrillar degeneration and subsarcolemnal collections of mitochondria were also noted. The mitochondria showed no abnormal features.

In the deltoid muscle biopsy, there was only mild variation in fibre size. Small rounded fibres mostly of type I, were randomly distributed (fig. 3). There was no fibre necrosis, degeneration or regeneration. Electromicroscopy showed a few fibres with focal myofibrillar degeneration and excess glycogen accummulation. In both biopsies, inflammatory infiltrates were not seen.

\section{Nerve biopsy}

There was no evidence of axonal loss, axonal degeneration, demyelination or remyelination on light microscopy and electronmicroscopy of both superficial and deep peroneal nerves. The density of myelinated fibres was within the normal range (table 1). The myelinated fibre diameter histograms showed a normal bimodal distribution (Fig 4C, D). Teased fibre analysis of the deep peroneal nerve was also normal (table 2).

\section{Discussion}

Both patients presented with clinical features consistent with the diagnosis of scapuloperoneal syndrome. Weakness and wasting occurred in a typical distribution and no known cause was determined. In both patients, as is not uncommon with the scapuloperoneal syndrome, neither the clinical examination nor the electrophysiological studies differentiated between a myopathic and a neurogenic disorder with certainty.

Clinically, the combination of proximal upper and distal lower limb wasting was associated with the loss of deep tendon reflexes. Nerve conduction studies demonstrated normal values in both patients. In patient 1 , features suggestive of a myopathy and of denervation were both present on EMG of distal leg muscles. In patient 2 , polyphasic motor unit potentials with increased duration, increased amplitude and increased firing rates would be more suggestive of chronic denervation. However, the diagnosis of a myopathic or neurogenic process could not be made solely on the basis of the EMG.

To determine the underlying basis of the syndrome, as to whether it is a myopathy, a neuropathy or a spinal muscular atrophy, selected multiple muscle and nerve biopsies were performed. Muscle biopsies were performed on a severely wasted distal leg muscle and a moderately affected proximal shoulder muscle. In patient 1 , specific features of neurogenic or myopathic atrophy could not be distinguished in the extensor hallucis longus muscle because of severe replacement by fat and connective tissue. In patient 2 , the distal leg muscle biopsy specimen showed features in favour of a myopathy, with random fibre size variation, hypertrophied fibres and rounded small fibres, internal nuclei and frequent ringbinden. In the deltoid muscles

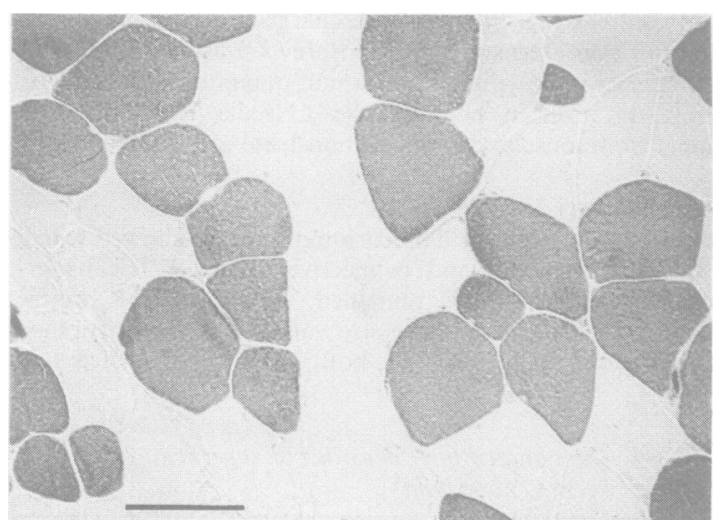

Fig 3 Deltoid, Case 2. Fibre size variation, randomly distributed fibre types. Small fibres mostly Type 1. ATPase stain ph 4.3. (Bar $=50 \mu \mathrm{m})$. 


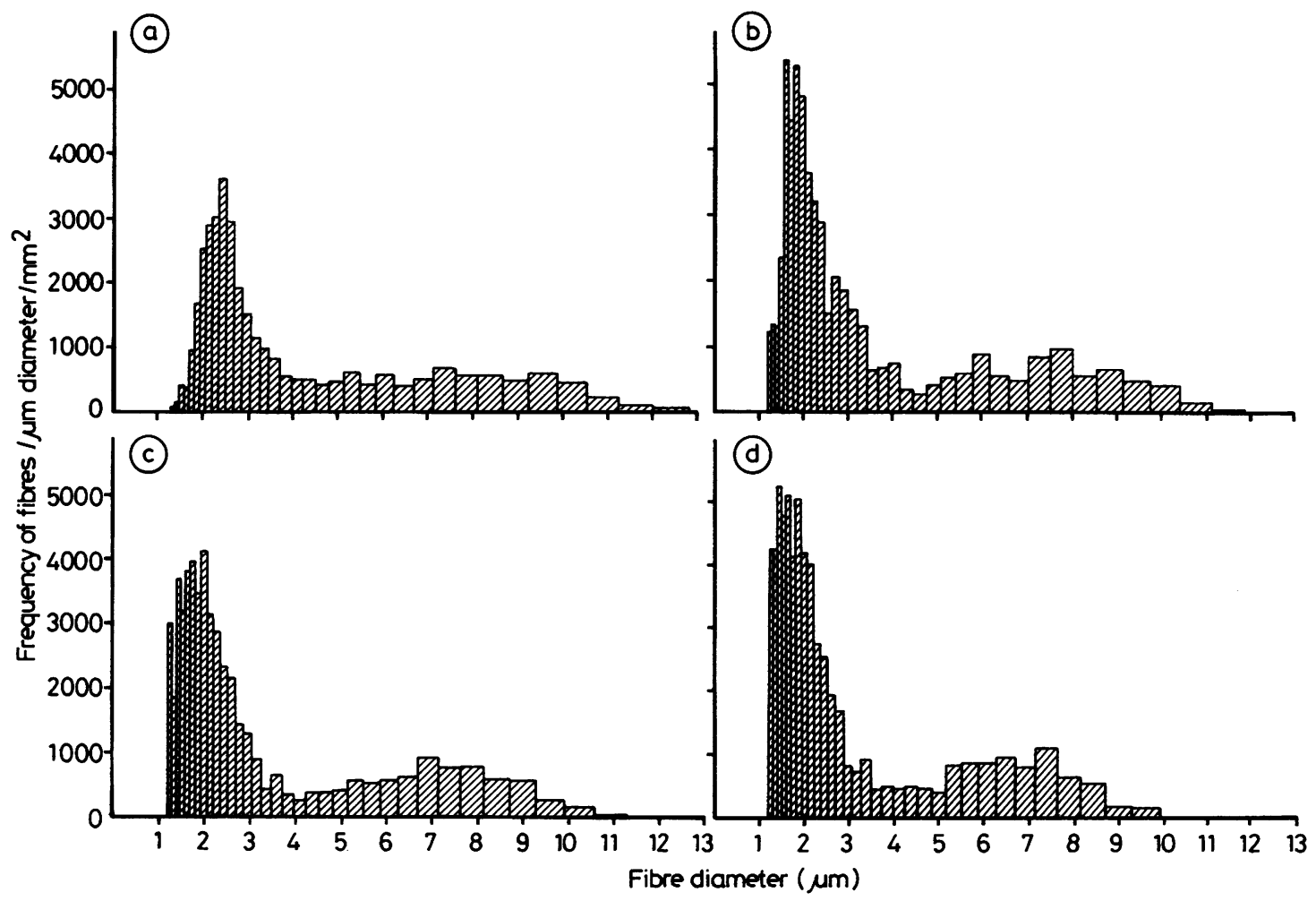

Fig 4 Myelinated fibre diameter distributions of superficial (SPN) and deep (DPN) peroneal nerves. (a-Case 1, DPN; $b-$ Case 1, SPN; $c-$ Case 2, DPN; $d$-Case 2, SPN).

of both patients, mild changes were seen, such as internal nuclei, random fibre size variation and atrophy, fibre splitting and ringbinden, which were suggestive of a myopathy. Specific features of neurogenic atrophy were lacking in these biopsies.

In order to resolve concerns of an underlying neurogenic disorder, biopsies were performed on the deep and superficial peroneal nerves. It should be noted that muscles supplied by the deep peroneal nerve included some severely wasted muscles. Careful examination of semithin sections by light microscopy and ultrathin sections by electron microscopy showed no evidence of chronic axonal loss, active axonal degeneration, demyelination or remyelination. These observations were further supported by normal myelinated fibre densities, diameter distributions and teased fibre analysis.

These normal nerve biopsy results taken in conjunction with the muscle biopsy findings rule out the likelihood of an underlying peripheral neuropathy or spinal muscular atrophy. We can conclude with certainty that these two patients have a primary myopathy in the category of scapuloperoneal myopathy.

The difficulty encountered in differentiating between a myopathic and neurogenic disorder in these two patients is not unusual for this syndrome, and is a recurrent theme in the literature. It is not possible to ascertain the exact nature of cases initially identified by Brossard ${ }^{9}$ and other early authors, prior to the advent of electromyography and muscle histochemistry. Subsequent reports have related their cases to either a peripheral neuropathy, ${ }^{10-13}$ a spinal muscular atrophy ${ }^{14-19}$ or a primary myopathy. ${ }^{20-25}$ These reports relied on EMG and nerve conduction studies and muscle biopsy histology, either alone or in combination, to diagnose the underlying disorder.

A number of reports have specifically commented on the problem of identifying a neurogenic or myopathic disorder in the individual patient with the scapuloperoneal syndrome. ${ }^{245}$ Feigenbaum and Munsat ${ }^{4}$ noted that EMGs were normal or indeterminate in three of their 10 cases. In one case, a "myopathic" EMG was associated with features of denervation on 
muscle biopsy. Carroll, ${ }^{2}$ in his review, illustrated this problem with a case showing similar discordance between EMG and muscle biopsy and other cases whose muscle biopsy showed non-specific or complex changes that were difficult to interpret. Indeed, cases that have been related to a myopathy in the literature not infrequently showed features on muscle biopsy that were suggestive but not specific for a myopathy. ${ }^{167}$ Some reported cases showed severe endstage changes in muscle that made differentiation between a myopathic and neurogenic cause difficult. $^{67}$ On the other hand, cases related to a neurogenic cause in the literature have also showed the coexistence of myopathic features on EMG or muscle biopsy. ${ }^{15-17}$ In the presence of these difficulties, in individual cases, such as the two patients reported here, extensive analysis of selective multiple muscle and nerve biopsies would resolve most doubts in the diagnosis.

The diagnosis of scapuloperoneal myopathy in these two patients requires further analysis as it is itself a heterogenous entity. Thus, a distinct group of patients with sex linked inheritance, multiple contractures, and cardiac dysrhythmia has been identified. ${ }^{26-29}$ Thomas et $a l^{6}$ distinguished an entity of adult onset scapuloperoneal myopathy. Of his six cases, two were sporadic, two of indeterminate inheritance and two showed autosomal dominant inheritance. Two additional cases with probable autosomal dominant inheritance were described by Todman and Cooke. ${ }^{7}$ Serratrice et al, ${ }^{1}$ in their review of 22 cases of "scapuloperonal myopathy", expressed the opinion that those cases with dominant inheritance were simple variants of facioscapulohumeral muscular dystrophy. Although some of these had late onset, most had younger ages of onset. Facial weakness was also common in this group. However, in their series, Serratrice et al regarded three sporadic cases, all with adult onset, as a distinct group, possibly related to the "restricted myopathies."

Our two cases clinically resemble the cases with adult onset described in these reports. As with other reported cases, specific abnormalities were not noted in muscle histology, although prominent ringbinden were an unusual feature in the muscle biopsy specimens of our cases. Both cases were sporadic, and their identification again suggests a distinct entity of adult onset scapuloperoneal myopathy, separate from facioscapulohumeral muscular dystrophy. It may be anticipated that linkage studies with specific genetic markers will help to resolve this controversy.

The authors thank David Mallott and Gordon Helps for their technical assistance and Debra Jones for assistance in the preparation of the manuscript.

\section{References}

1 Serratrice G, Pellissier J-F, Cremiux G, et al. Scapuloperoneal myopathies, myelopathies, and neuropathies. In: Serratrice $\mathbf{G}$, Roux H, eds. Peroneal Atrophies and Related Disorders. New York: Masson, 1979;233-52.

2 Carroll JE. Facioscapulohumeral and scapuloperoneal syndromes. In: Vinken PJ, Bruyn GW, eds. Handbook of Clinical Neurology 1979;40:415-31.

3 Munsat TL. Facioscapulohumeral dystrophy and the scapuloperoneal syndrome. In: Engel AG, Banker BQ, eds. Myology 1986;2:1251-66.

4 Feigenbaum JA, Munsat TL. A neuromuscular syndrome of scapuloperoneal distribution. Bull Los Angeles Neurological Society 1970;35:47-57.

5 Bethlem J. The scapuloperoneal syndrome. In: Serratrice G, Roux $\mathrm{H}$, eds. Peroneal Atrophies and Related Disorders. New York: Masson, 1979;225-31.

6 Thomas PK, Schott GD, Morgan-Hughes JA. Adult onset scapuloperoneal myopathy. J Neurol Neurosurg Psychiatry 1975; 38:1008-15.

7 Todman DH, Cooke RA. Scapuloperoneal myopathy. Clin Exp Neurol 1984;20:169-74.

8 Dyck PJ, Karnes J, Lois A, Lofgren EP, Clark Stevens J. Pathological alterations of the peripheral nervous system of humans. In: Dyck PJ, Thomas PK, Lambert EH, Bunge R, eds. Peripheral Neuropathy 1984; Vol. I: 760-870.

9 Brossard J. Etude clinique sur forme héréditaire d'atrophie musculaire progressive débutant par les membres inférieurs (type femoral avec griffe des orteils). Thesis: Steinheil, Paris. 1886.

10 Davidenkow S. Scapulo-peroneal amyotrophy. Arch Neurol Psychiatry 1939;41:694-701.

11 Harding AE, Thomas PK. Distal and scapular peroneal distribue tions of muscle involvement occurring within a family with type I hereditary motor and sensory neuropathy. $J$ Neurof 1980;224:17-23.

12 Meadows J, Marsden CD. Scapulo-peroneal amyotrophy. Arch Neurol 1969;20:9-12.

13 Schwartz MS, Swash M. Scapuloperoneal atrophy with sensorg involvement: Davidenkow's syndrome. $J$ Neurol Neurosurg Psychiatry 1975;38:1063-7.

14 Emery ES, Fenichel CM, Eng G. A spinal muscular atrophy with scapulo-peroneal distribution. Arch Neurol 1968;18:129-33.

15 Kaeser HW. Scapuloperoneal muscular atrophy. Brain 1965; 88:407-18.

16 Mercelis R, Demeester J, Martin JJ. Neurogenic scapuloperoneal syndrome in childhood. J Neurol Neurosurg Psychiatry 1980; 43:888-96.

17 Ricker K, Mertens HG, Schimrich K. The neurogenic scapuloperoneal syndrome. Eur Neurol 1968;1:257-74.

18 Takahashi K, Nakamura H, Nakashima R. Scapuloperoneal dystrophy associated with neurogenic changes. $J$ Neurol Sci 1974;23:575-83.

19 Zellweger H, McCormick WF. Scapuloperoneal dystrophy and scapuloperoneal atrophy. Helvetica Paediatrica Acta 1968; 6:643-9.

20 Hausmanowa-Petrusewicz I, Zielinska S. Zur nosologischen stellung des scapulo-peronealen syndroms. Disch $Z$ Nervenheilk 1962;183:377-82.

21 Kazakov UM, Bogorodinsky DK, Skorometz AA. Myogenic scapuloperoneal syndrome-muscular dystrophy in the K Kindred. Eur Neurol 1975;13:350-9.

22 Lovelace RE, Menken M. The scapuloperoneal syndrome: dystrophic type. 4th International Congress on Neurological Surgery/9th International Congress on Neurology, N.Y. Amsterdam: Excerpta Medica 1969;193:247-8.

23 Ricker K, Mertens HG. The differential diagnosis of the myogenic-(facio)-scapulo-peroneal syndrome. Eur Neurol 1968;1:275-307.

24 Seitz D. Zur nosologischen stellung des sogennanten scap- 
uloperonealen syndroms. Dtsch Z Nervenheilk 1957;175 $547-52$.

25 Serratrice G, Roux H, Aquaron R, Gambarelli D, Baret J. Myopathies scapulo-peronières (á propos de 14 observations dont 8 avec atteinte faciale). Semaine des Hôpitaux (Paris) 1969:45:2678-83.

26 Rotthauwe HW, Mortier W, Beyer $\mathrm{H}$. Neuer typ einer recessiv $\mathrm{x}$-chromosomal verebten muskeldystrophie scapulo-humerodistal muskeldystrophie mit fruhzeitigen kontrakturen und herzrhythmusstorungen. Humangenetik 1972;16:181-200.

27 Rowland LP, Fetell M, Orarte M, Hays A, Singh N, Warrat FE. Emery-Dreifuss muscular dystrophy. Ann Neurol 1979; 5:111-7.

28 Thomas PK, Calne DB, Elliott CF. X-linked scapuloperoneal syndrome. J Neurol Neurosurg Psychiatry 1972;35:208-15.

29 Emery AE, Dreifuss FE. Unusual type of benign $x$-linked muscular dystrophy. J Neurol Neurosurg Psychiatry 1966;29:338-42. 